\title{
GeoFairy: Towards a One-stop and Location Based Service for Geospatial Information Retrieval
}

\begin{abstract}
It is still a great challenge to efficiently deliver dynamic and heterogeneous Earth observation (EO) information to users based on their real time locations. However, the rapidly evolving techniques create a chance to meet the challenge. This paper proposes a framework to realize a one-stop and location based service (LBS) for geospatial information (GI) retrieval on mobile platforms. The framework originally integrates a number of state-of-the-art techniques with geospatial data resources and let them cooperate together to provide a robust and highly available LBS. Cloud platform is used to deploy the server module. A location enabled load balancing algorithm is presented to balance the cloud instance VMs on behalf of LBS requirements. A system named GeoFairy is implemented. It provides a one-stop service for gathering and delivering twelve kinds of GI on real time locations. Two Apps are built for the two major mobile ecosystems: iOS and Android. Many tests, including a stress test, have been made via a number of mobile devices at various locations. The results demonstrate that GeoFairy is capable of one-stop delivering real-time GI to users and significantly reducing costs on information searching and retrieving. This feature is very helpful in many scenarios such as disaster responding and military actions. This research paves the way on both theoretical and practical aspects for researchers and developers to realize operational mobile applications for one stop and location based GI retrieval.
\end{abstract}

Keywords: one-stop service; LBS; geospatial web service; geospatial cloud; geospatial mobile application.

\section{Introduction}

Mobile devices play an essential role in our lives today. Since wireless network is turned on by a great amount of devices, mobile platforms have already become the mainstream method for people to search and retrieve information. Location based services are widely deployed as mobile Apps to customize and deliver various kinds of information and capabilities to users.

A number of such Apps are already on the shelves, e.g. Google Maps, Apple Maps, Navigator, The Weather Channel, AccuWeather, Climate FieldView and ESRI ArcGIS mobile. Each App is designed to provide some specific information in a relatively static way, e.g., Google Maps for maps and satellite images, AccuWeather 
for current weather and weather forecast, Climate FieldView for agricultural field related information and ArcGIS for spatial data viewing and analyzing. The Apps have different transfer channels and separated outlets. Users have to download and install an App to acquire the contained information at one time. Besides, extra operations are often needed such as signing up, subscribing services, learning user guide and formalizing recognizable requests. It is complicated and very inconvenient for most users. A one-stop simplified App serving all kinds of GI has been widely recognized as a public desire, especially in emergent scenarios like response actions to disasters like earthquakes, flooding, wildfires and hurricanes. However, there are very few progresses towards this direction yet. The main challenge comes from the high heterogeneity and poor interoperability of the involved data and service interfaces.

As plenty of EO datasets have been released by agencies such as NASA (National Aeronautics and Space Administration), NOAA (National Oceanic and Atmospheric Administration), USGS (U.S. Geological Survey), ESA (European Space Agency) and EEA (European Environment Agency), the first problem becomes how to collect and decode those products, extract and encode information, and display it on an intuitive interface. To ensure the sources are live update, the App is required to real-time and on-demand retrieve data from providers via machine-to-machine communication. During this process, interoperability issues are inevitably raised. Protocols and standards are used to facilitate the communication. Standard interfaces can formalize the access, negotiation and delivery among clients and services. There are a number of standards which have been established by several organizations like OGC (Open Geospatial Consortium), ISO (International Organization for Standardization), W3C (World Wide Web Consortium) and OASIS (Organization for the Advancement of Structured Information Standards) and widely applied in industrial businesses. Most of the existing services are standard compliant and demand clients to learn about those standards first before using them. Therefore, it is a big challenge to realize the ability automatically interacting with disparate service standards and data formats, and turn on the intelligent collection and stream from geospatial data providers to endpoint devices.

To address these issues, this paper proposes a LBS framework for one stop retrieval of GI on mobile platforms. The framework integrates a number of state-of-the-art techniques with geospatial resources and let them cooperate together to provide a robust and highly available LBS. A system named GeoFairy is implemented. It supports to gather and deliver twelve kinds of geospatial related information on real time user locations. Two clients are built to adapt to the iOS and Android systems. GeoFairy is preliminarily tested on twenty six phones at various 
locations. The results show that GeoFairy is capable of one-stop delivering multiple GI of current locations to consumers and reducing cost on information searching and retrieving. This capability is extremely helpful in scenarios like agricultural activity planning, vegetation monitoring, disaster response and daily inquiry. On both practical and theoretical aspects, GeoFairy sets an example to the community in realizing one-stop operational LBS for GI distribution and retrieval.

The remainder of the paper is organized as follows. Section 2 introduces the background of this work. Section 3 investigates the related work. Section 4 describes the proposed framework. Section 5 briefs the implementation of GeoFairy. In Section 6 , tests are made on GeoFairy and the results are evaluated. Section 7 discusses the advantages and disadvantages of GeoFairy. The conclusion and future work are given in Section 8.

\section{Background}

The state-of-the-art technologies create a suitable environment for the born of GeoFairy. Fig. 1 shows the related architectures and platforms. Wireless network makes mobile devices accessible to the Internet. Mobile software development kit (SDK) is used to write mobile Apps. Geospatial web services provide geospatial data products and processing capabilities on the Web. RESTful services support concise interfaces for clients. LBS customizes the service content according to coordinates. High performance computing (HPC) can supply powerful server side computation capability.

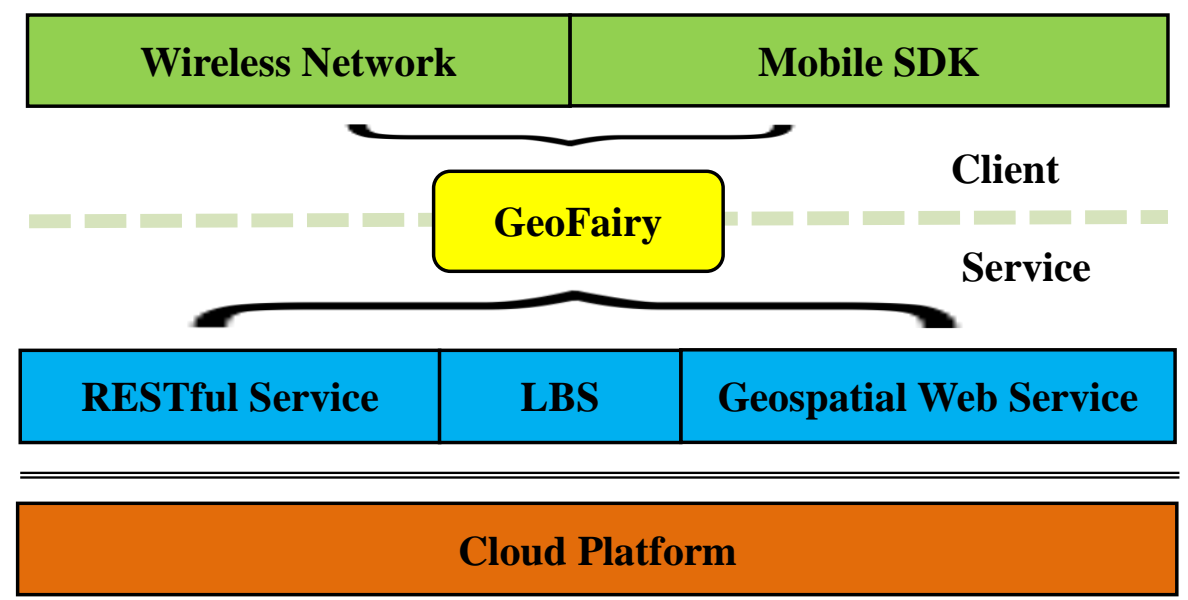

Figure 1. The relationships between GeoFairy and the state-of-the-art technologies

\subsection{Wireless network}


Wireless network uses radio and spectrum to conduct data communication among devices [1]. Two common techniques are Wi-Fi and cellular network [2]. The former represents "wireless local area network" (WLAN) from IEEE (Institute of Electrical and Electronics Engineers) 802.11 standards [3, 4]. The latter divides the region into areas, also called cells [5-7]. Each cell is configured with a fixed cell station to receive or relay radio waves and transfer signals to or from mobile devices. Wireless network removes spatial constraint on the endpoint devices and make them able to connect into the Internet anytime and anywhere. As the cost of WIFI and Cellular network is relatively lower than other wireless network, GeoFairy uses them to transfer the messages between client and server module.

\subsection{Mobile SDK}

Comparing to old phones, smart phones have more functionality besides making calls [8], e.g. checking weather forecast, searching restaurants, navigation, taking photos, recording videos and surfing on social media. Mobile operating systems have gone through a very competitive race and evolved to two major platforms: Google Android and Apple iOS. Both provide a set of development tools to attract developers to contribute their ideas and applications. They also offer APIs to be invoked by outside programs. These tools are usually called SDK (software development kit), e.g. Android SDK and iOS SDK (Xcode). There are also some other alternative SDKs like Corona SDK [9] and Apache Cordova SDK [10, 11]. Apache Cordova is an open source and cross-platform mobile development framework. It uses standard web techniques, HTML5, CSS3 and Javascript and automatically makes them available on all the platforms without duplicated development. Due to its cross platform, low maintenance cost and easy-to-transplant feature, Apache Cordova is adopted in this research.

\subsection{Cyberinfrastructure and geospatial web services}

Cyberinfrastructure (CI) is used by United States NSF (National Science Foundation) to describe a technical solution for connecting data, software, computers, storage, processors and people together to treat sophisticated multidisciplinary problems $[12,13]$. Specific CIs for geospatial domain are later addressed as CyberGIS [14], spatial CI [15] or geospatial CI [16]. In all the physical frameworks of geospatial $\mathrm{CI}$, the core component is consistently geospatial web service. Geospatial web services are built to publish local resources online so that users can remotely access them. The server capabilities include retrieving, processing, analyzing and visualizing geospatial data. Standardized interfaces are needed to improve interoperability. There are several international service standardization organizations. Standards, such as 
SOAP (Simple Object Access Protocol) WSDL (Web Service Definition Language), WMS (Web Map Service), WCS (Web Coverage Service), WFS (Web Feature Service), WPS (Web Processing Service), CSW (Catalogue Service), SOS (Sensor Observation Service) and SPS (Sensor Planning Service), have been established and widely adopted in both the industrial and academic communities. A series of open source libraries, tools and software have been released to facilitate the implementation of web services and clients compliant to these standards, like GeoServer, MapServer, pyCSW, pyWPS, EoxServer, 52 ${ }^{\circ}$ North, Quantum GIS, GRASS GIS, CartoWeb, ka-Map, Mapbender, OpenLayers, etc [17]. Therefore, many formal geospatial web services are available like MODAPS WCS [18], NASA GES DISC WCS [19] and NASA NEO WMS [20]. Instead of developing new services which are uneconomic and unnecessary, GeoFairy reuses the existing services to retrieve all the needed GI.

\subsection{RESTful services}

REST stands for REpresentational State Transfer and is a style for architecting large-scale distributed systems [21]. Its idea depends on stateless client-to-server communication above web transfer protocol. In most cases, HTTP (Hypertext Transfer Protocol) is the protocol. RESTful services use URI (Uniform Resource Identifier) to identify resources, have uniform interface (GET, POST, DELETE, PUT) and self-descriptive messages, and conduct stateful interaction via hyperlinks. The messages are mostly in JSON (JavaScript Object Notation) which is more lightweight than XML. The architecture gives a concise pattern for publishing and consuming web services and quickly draws interests from the public [22]. Many RESTful services have been developed for basic and ad-hoc geospatial requirements [23]. The standard groups are trying to include RESTful interface into their standards [24]. GeoFairy also takes advantage of RESTful services.

\subsection{LBS}

LBS uses coordinates of mobile devices to provide customized service content. LBS leverages hardware locator to tracking real time location. The locator integrates various technologies such as GPS (global positioning service) [25], GSM (Global System for Mobile communications) localization [26] and Indoor Positioning technique [27]. Typical LBS includes intelligent navigation, location-based advertising, nearby business recommendation, local social network, etc [28-30]. GIS (geographic information system) and RS (remote sensing) researchers keep trying to bind geospatial data and functions to LBS [31,32]. In this paper, GeoFairy aims to provide a real-time LBS serving multiple local GI at one time. 


\subsection{Cloud computing}

Among HPC techniques, cloud computing is the most popular one nowadays. It builds a platform with scalable and virtualized resources that can efficiently serve tens of thousands of people at the same time [33]. In a widely accepted definition [34], cloud computing is explained as:

"Cloud computing is a model for enabling ubiquitous, convenient, on-demand network access to a shared pool of configurable computing resources (e.g., networks, servers, storage, applications, and services) that can be rapidly provisioned and released with minimal management effort or service provider interaction. "

The model is composed of three service models (Software as a Service (SaaS), Platform as a Service (PaaS) and Infrastructure as a Service (IaaS)), and four deployment models (Private Cloud, Community Cloud, Public Cloud and Hybrid Cloud) [34]. Each model has at least one implementation. For example, the open source software for IaaS has OpenStack [35], CloudStack [36], Eucalyptus [37] and OpenNebula [38]. Open source PaaS software includes Cloud Foundry, Cloudify, OpenShift, Stackato and Apache Stratos. Using the software, cloud platforms with different size, scale and capabilities are built by various vendors such as Google, Amazon, Microsoft and numerous small and medium enterprises (SME).

GeoFairy is benefited by cloud computing to become more elastic and flexible. Its service module is deployed on instances of a private cloud platform created by Apache CloudStack. A load balancer is configured to assign the load on particular instances. CloudStack provides a command line interface (CloudStack-CLI) to start or shut instances. Based on CloudStack-CLI, GeoFairy embeds a self-adaptive mechanism to adjust the number of instances according to the overall user traffic. When the traffic is low, GeoFairy will shut down a few instances and release the occupied resources. If the traffic is high, new instances will be started to keep a reasonable load on each instance. Other HPC techniques such as grid computing and distributed computing have limited libraries and require heavy programming work and are less suitable for GeoFairy.

\section{Related Work}

An investigation has been conducted on the recent researches of one-stop geospatial services. Geoportal represents a website as one-stop entry point to GI. Tait reviewed four key distributed GIS portal projects in 2004 and discussed the challenges for wider usage of GIS [39]. Maguire et al studied the emergence of 
geoportals and discussed the contribution of SDI (spatial data infrastructure) to simplifying the access to GI [40]. Goodchild et al reviewed the history of geospatial data products and introduced the GOS (Geospatial One-Stop) which aims to provide a single portal to geographic information [41]. The GOS is comprised of a Web portal, a catalog of FGDC (Federal Geographic Data Committee) metadata and some services for validation and harvest. Bernard et al also introduced the European Geoportal as one building block of the ESDI (European Spatial Data Infrastructure) [42]. Giuliani et al developed the PREVIEW Global Risk Data Platform as one-stop geoportal to more than sixty datasets about nine natural hazards [43]. Roche et al realized a portal to produce and spread geographic information on behalf of crisis management [44]. Croner highlighted the benefits of web-accessible one-stop geoportal with georeferenced public health information on advance disease surveillance, control, and prevention [45].

As for LBS, the existing work focuses on Apps for searching traditional business information (restaurants, hospital, gas station, etc) and local news [46-48]. Chuang et al introduced how to enable map based search for business information on mobile devices via focused crawling and information extraction [49]. Becker et al described a location-aware App named DBpedia Mobile which can indicate the nearby interest locations from DBpedia on a map [50]. Yin et al developed Touch2Query allowing users to draw shapes on touch screen as AOI (Area of Interest) to perform ad-hoc spatial searches [51]. Bao et al created a location based and preference aware recommendation system using geo-social networking data [52]. In methodology, Abdalla clarified the differences and relationships between mobile GIS and LBS and the challenges facing by mobile GIS like limited bandwidth, battery life, small screen size, high cost of networks, and multiple, often conflicting standards [53].

However, the geoportals provide only links and are not location oriented. When users find the desired records in the portals, they are usually redirected to some other websites to retrieve the original entities. Thus they are not strictly one-stop. The portals are also lack of mobile clients to take users' real time locations into account. On the aspect of LBS, there is no such a service yet to support one-stop and real time location based retrieval of multiple GI. The challenges include the physical limits of mobile devices and the heterogeneity of GI sources. GeoFairy is proposed and implemented to overcome these obstacles.

\section{Framework}

This section introduces a client-and-server framework (shown in Fig. 2). The 
service module is divided into several sub modules: request handler, data collector, service adapter, data decoder, data cache, information encoder and load balancer. The client module includes five sub modules: location manager, information viewer, request builder, response parser and message transceiver. All the modules are explained below.

\subsection{Server Module}

The server module aims to collect geospatial data and send information in concise format back to clients. The modules are loosely coupled and can be deployed on any operating systems. The framework supports multiple instance virtual machines (VM) running in parallel. A load balancer is configured to balance the workload on each VM.

1) Request handler is used to directly deal with the received requests and send back information. This module is responsible for understanding user intentions by learning request content and invoking respective responders in data collector to process the request. Meantime, as the only entry to the whole server module, request handler is responsible for distinguishing legal requests from illegal ones and protecting the server from cyber-attacks and unintentional Internet traffics.

2) Data collector is used to collect data from various online sources. Data need be retrieved from the services in Table 1 . The services are wrapped by different protocols and interfaces. Those products are either measured in fields or simulated by models. The collection is a heavy duty which costs many system resources. A good standing server with fast network is required to complete each collection job. Once received an order from request handler, data collector will initiate a responder to process the order. The responder will be loaded with a service adapter to generate standard compliant requests, as only legally formatted requests can get correct responses from the services. For example, OGC services accept XML based requests formatted by a number of OGC XSDs (XML Schema Definition). WSDL enabled services communicate with clients via SOAP messages. RESTful services accept GET and POST requests and return JSON. Service adapters automatically compose legal requests out of the collection requests and send them to the target services. 
Service Providers

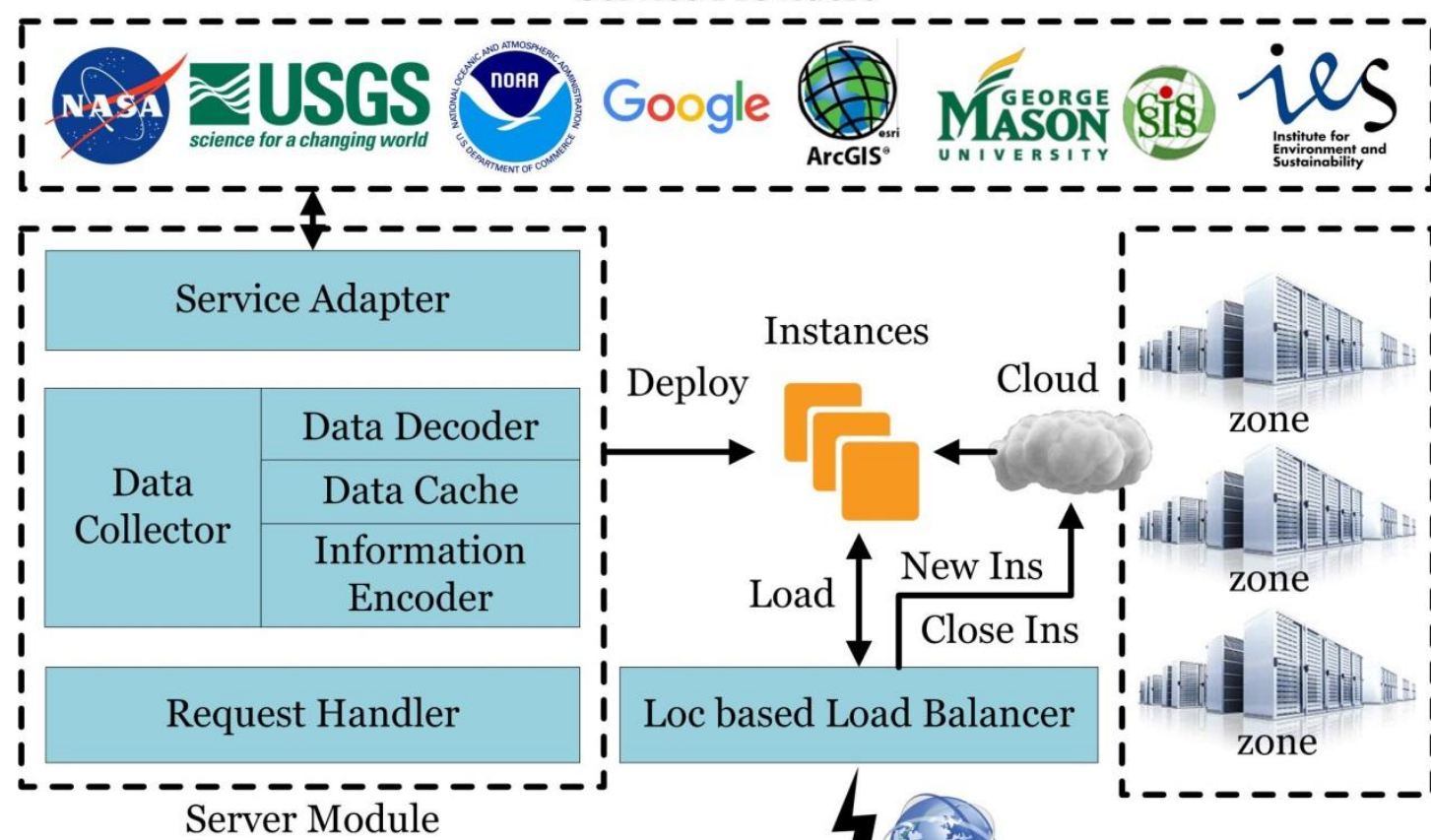

Client Module

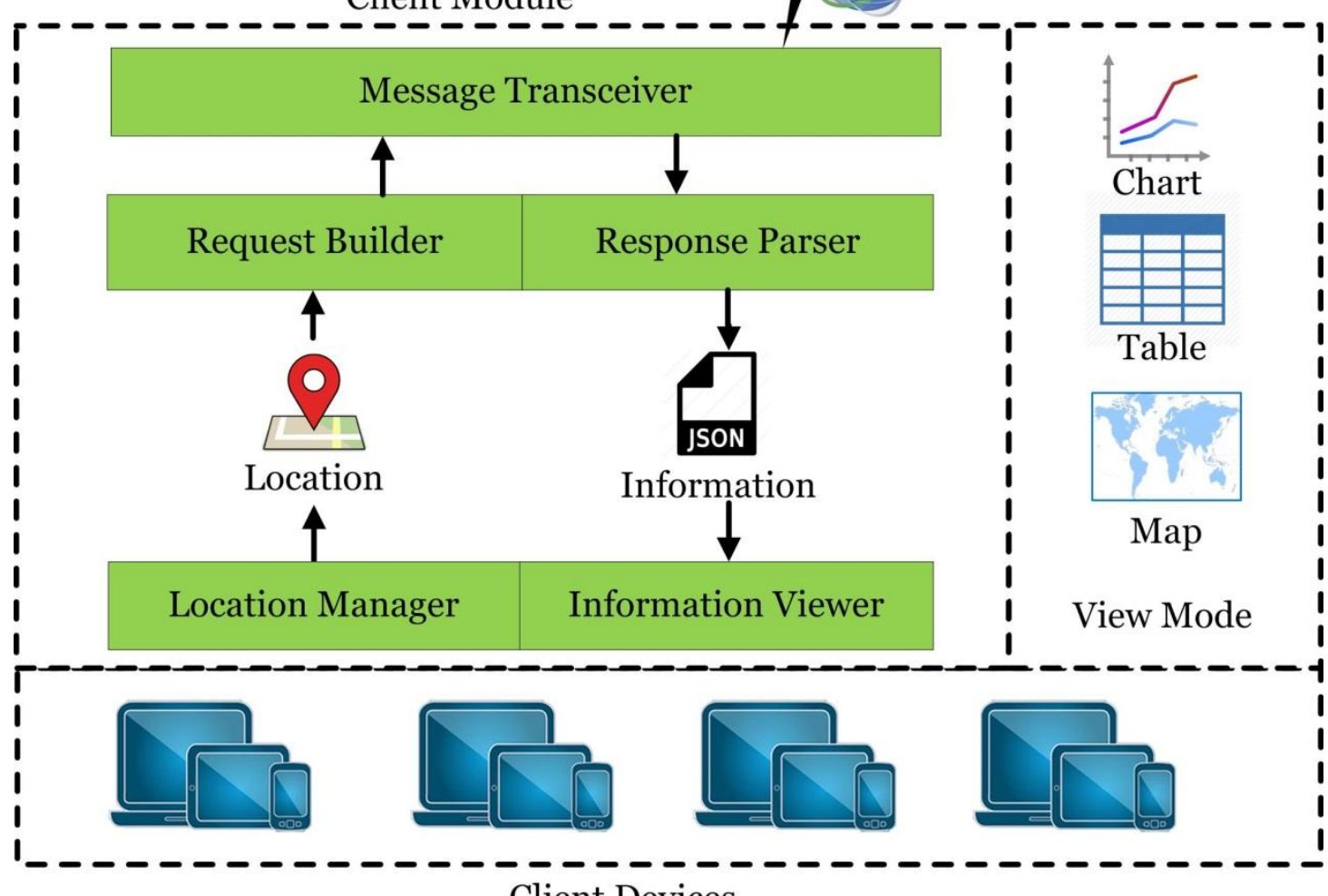

Client Devices

Figure 2. The framework of GeoFairy

Table 1. A list of the web services providing geospatial data products

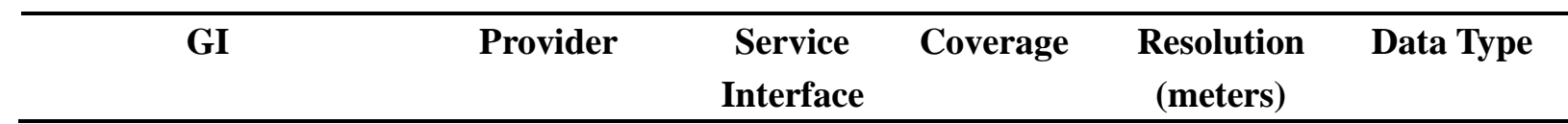




\begin{tabular}{|c|c|c|c|c|c|}
\hline Location Address & Google & REST & Globe & - & Literal \\
\hline Latitude/Longitude & Google & REST & Globe & - & Literal \\
\hline Global Vector Map & USGS & HTTP & Globe & - & Vector \\
\hline Elevation & USGS & WMS & Globe & 30 & Raster \\
\hline Weather \& Forecast & $\begin{array}{c}\text { NOAA/ } \\
\text { OpenWeatherMap }\end{array}$ & REST & $\begin{array}{l}\text { U.S./ } \\
\text { Globe }\end{array}$ & - & Literal \\
\hline Vegetation & GMU & WMS & Globe & 250 & Raster \\
\hline Soil Moisture & NOAA & REST & Globe & 400 & Raster \\
\hline Atmosphere & NOAA & WMS & Globe & 1,300 & Raster \\
\hline Global Land Cover & JRC-IES & REST & Globe & 1,000 & Raster \\
\hline Cropland Data Layer & USDA & WMS & U.S. & 30 & Raster \\
\hline Agriculture Hardiness & USDA & REST & U.S. & - & Literal \\
\hline Precipitation & NOAA & WMS & Globe & 1,300 & Raster \\
\hline $\begin{array}{l}\text { USGS: United States G } \\
\text { NOAA: National Ocea } \\
\text { GMU: George Mason } \\
\text { NASA: National Aeron } \\
\text { JRC-IES: Institute for } \\
\text { Commission's Joint Re } \\
\text { USDA: United States }\end{array}$ & $\begin{array}{l}\text { eological Survey } \\
\text { ic and Atmospheric } A \\
\text { niversity } \\
\text { autics and Space Adm } \\
\text { Environment and S } \\
\text { earch Centre } \\
\text { epartment of Agricult }\end{array}$ & $\begin{array}{l}\text { ministrat } \\
\text { istration } \\
\text { tainabilit }\end{array}$ & Europe & & \\
\hline
\end{tabular}

3) Service adapter is used to translate data requirements to standardized messages and unwrap the information from response messages. It is based on the schema of standards and able to compose legal service requests, embed user constraints like bounding box and temporal period into appropriate elements and parse service response messages to extract the content or data links.

4) Data decoder is used to decode the data returned by service adapter into information. The retrieved data from service adapter is usually encoded by some kind of formats such as GeoTiff, HDF (Hierarchical Data Format), ESRI Shapefile and GeoJSON. They have to be decoded to expose the contained information which is usually number with unit (e.g., temperature, humidity, NDVI (Normalized Difference Vegetation Index), land cover code, hardiness zone code, etc). Data decoder adopts geospatial data I/O (input/output) libraries and outputs the extracted information. The resolution inconsistency problem exists on both spatial and temporal aspects when heterogeneous data sources are involved. For raster data, no matter what the spatial resolution is, the value of the pixel where the coordinate locates will be considered as the location's value. For vector data, the property value of the polygon containing the location will be used. Temporal resolution inconsistency is more serious for many data products. Commonly, the latest product will be used. Sometimes, historical records will be all listed for users to compare the changes in the past years, e.g. cropland data layer (from 2008 to 2015). 
5) Information encoder is used to encode the information into some concise format such as JSON. The information from data decoder is in plain text and need be encoded to facilitate the interaction between server and client. JSON is used as the encoding format as it is easier for clients to parse and understand. An example NDVI JSON is displayed in Table 2. The encoded information will be delivered to request handler. Request handler will transfer it back to the initiative client module.

Table 2. An example NDVI JSON

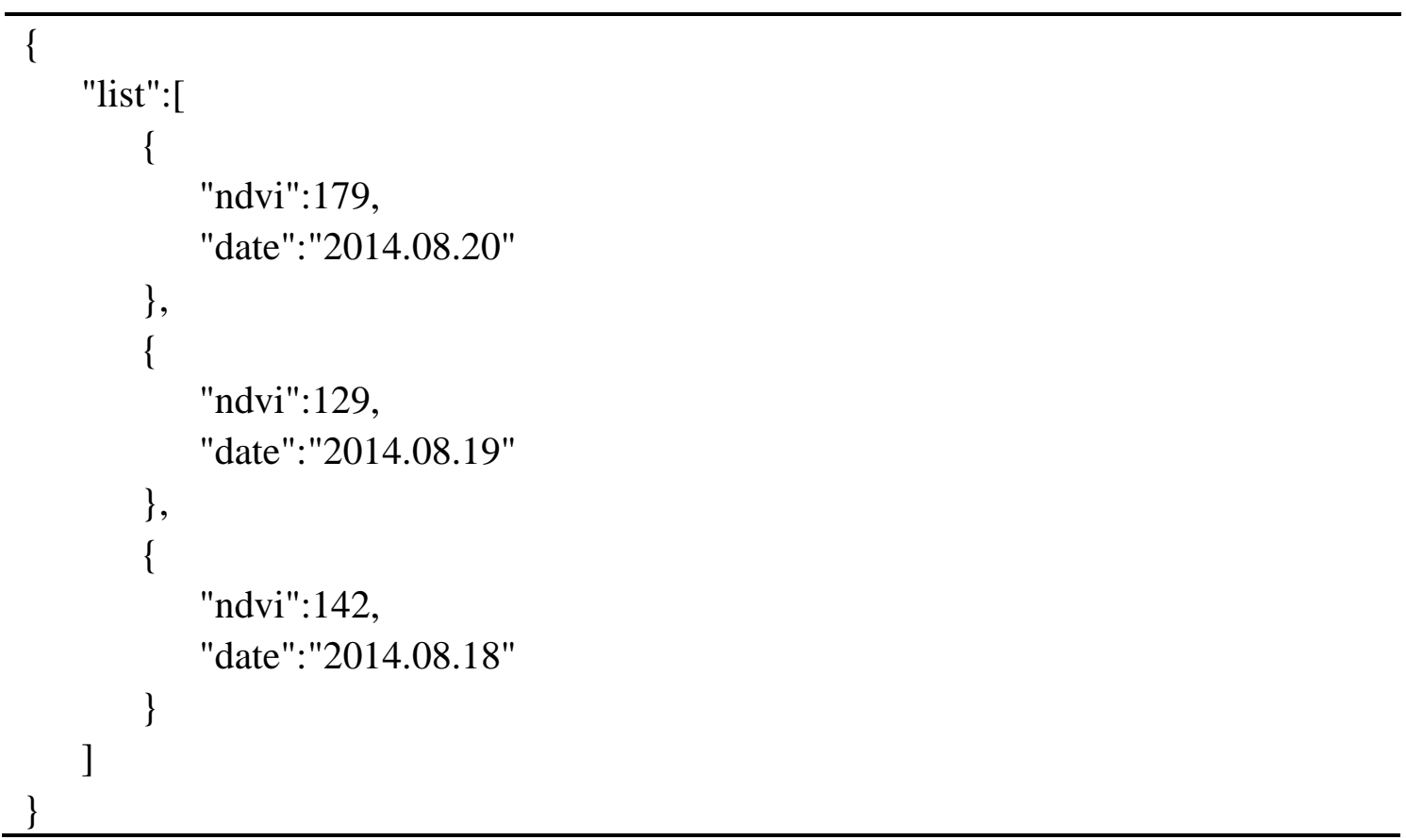

6) Data cache is used to cache the data captured from remote providers. Because the services are distributed and their network conditions vary, frequent connections to the services will cost longer waiting time on message delivery. A cache mechanism will significantly reduce the connecting times between GeoFairy and remote services. As shown in Fig. 3, the data cache module takes location as input and makes a judgement via cache manager. The manager tells whether the data for the location is cached. If cached, it simply returns the data to users. If not, the requests are transferred to service adapter for actual contact with the remote services. The retrieved data will be returned to endpoint users and cached at the same time. There are three levels of cache in GeoFairy: point, box and map. Point cache records a report of a coordinate. Box cache records the imagery of a bounding box. Map scale cache stores the data within the whole map area. Commonly, point cache is used for SOAP and RESTful services which provide values for specific coordinates. For other services like WCS, WFS and WMS which are unable to simply return the information of a coordinate, service adapter will retrieve the data of a bounding box containing the location. The data is later cached as a box cache. Map cache is generated while users switch to the 
map view mode. All the data within the map area will be cached to accelerate the loading speed.

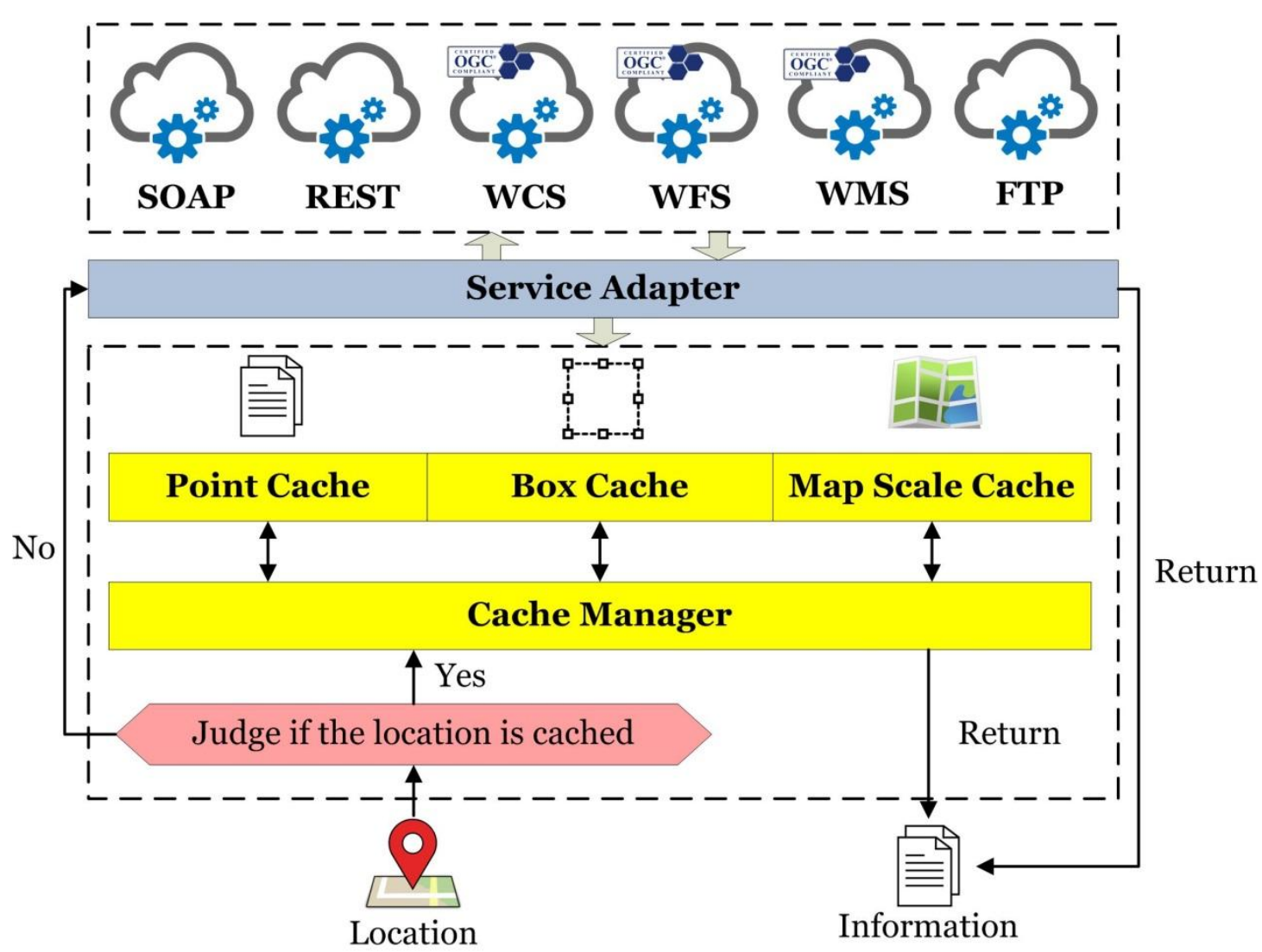

Figure 3. Data cache mechanism of GeoFairy

7) Location enabled load balancer is used to balance the load on instance VMs. As shown in Fig. 4, besides the traditional factors like request volume, live connections, source IPs and LRT (lowest response time), GeoFairy load balancer also considers user location as an impact factor. A major reason is that the data cache varies on different instances. Location enabled balancer tries to insure the instance with corresponding data cache will process the requests. The commonly used balancing strategies include round robin, least connections and IP harsh. We add location as new priority index into these strategies. For example, we use weighted round robin in our implementation. The algorithm is defined as:

$$
\begin{gathered}
W(i)=\text { Capa }(i)+\text { LocationCacheWeight }(i, x) \\
\text { LocationCacheWeight }(i, x)=\left\{\begin{array}{c}
5, \text { if } i \text { has cache for } x \\
0, \text { if } i \text { doesn't have cache for } x
\end{array}\right.
\end{gathered}
$$

where $\mathrm{i}$ is an instance server and $\mathrm{x}$ is a location. $\mathrm{W}(i)$ represents the weight of $\mathrm{i}$ in the weighted round robin algorithm. Capa( $i)$ reflects the capacity of i. If server 1's capacity is two times more than server 2's, then Capa(1) will be 2 and Capa(2) 
will be 1. LocationCacheWeight $(i, x)$ is the added weight factor which is five when server $\mathrm{i}$ has cache for $\mathrm{x}$ and zero when i doesn't. Five is a reasonable number to balance between server capacity and location cache. It can also be changed to any other numbers in particular scenarios. The instances with corresponding cache will have heavier weights in the calculation and are more likely to be assigned with the tasks than the instances without cache. Also, load balancer is responsible for managing the instance VMs used by GeoFairy. CloudStack-CLI is called to start and shut instances. Once the traffic is low, it will stop assigning tasks to some instances and shut them down after they finished all the running tasks. If the traffic becomes high, it will start new instances to process the newly arrived tasks and maintain a reasonable average response time on behalf of endpoint consumers.

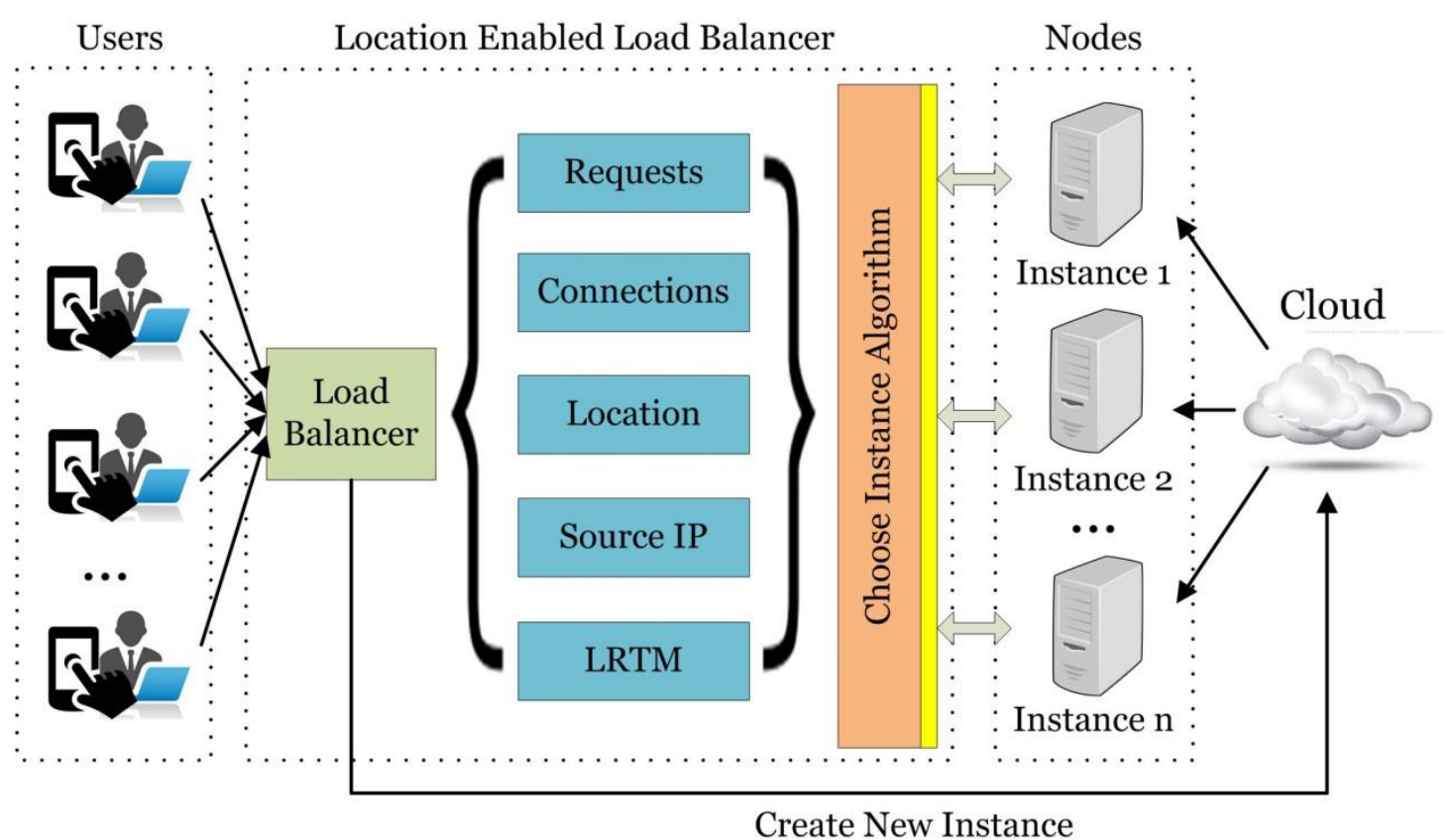

Figure 4. Location enabled load balancing mechanism

\subsection{Client Module}

Client module, particularly mobile Apps, is used to receive location based requests, interact with server module to retrieve information and display the information to users.

1) Information viewer is used to display GI to users. There are three major ways: table, chart and map. Table mode is suitable for checking the accurate numbers of GI. Chart mode is better when analyzing the trends and changes. Map mode overlays information and data layers on a basic map and gives users an intuitive understanding about the ongoing geospatial phenomenon. The same information can be displayed in 
multiple modes to better assist users in unscrambling the information easily and quickly. Fig. 5 demonstrates example interfaces of the three modes.
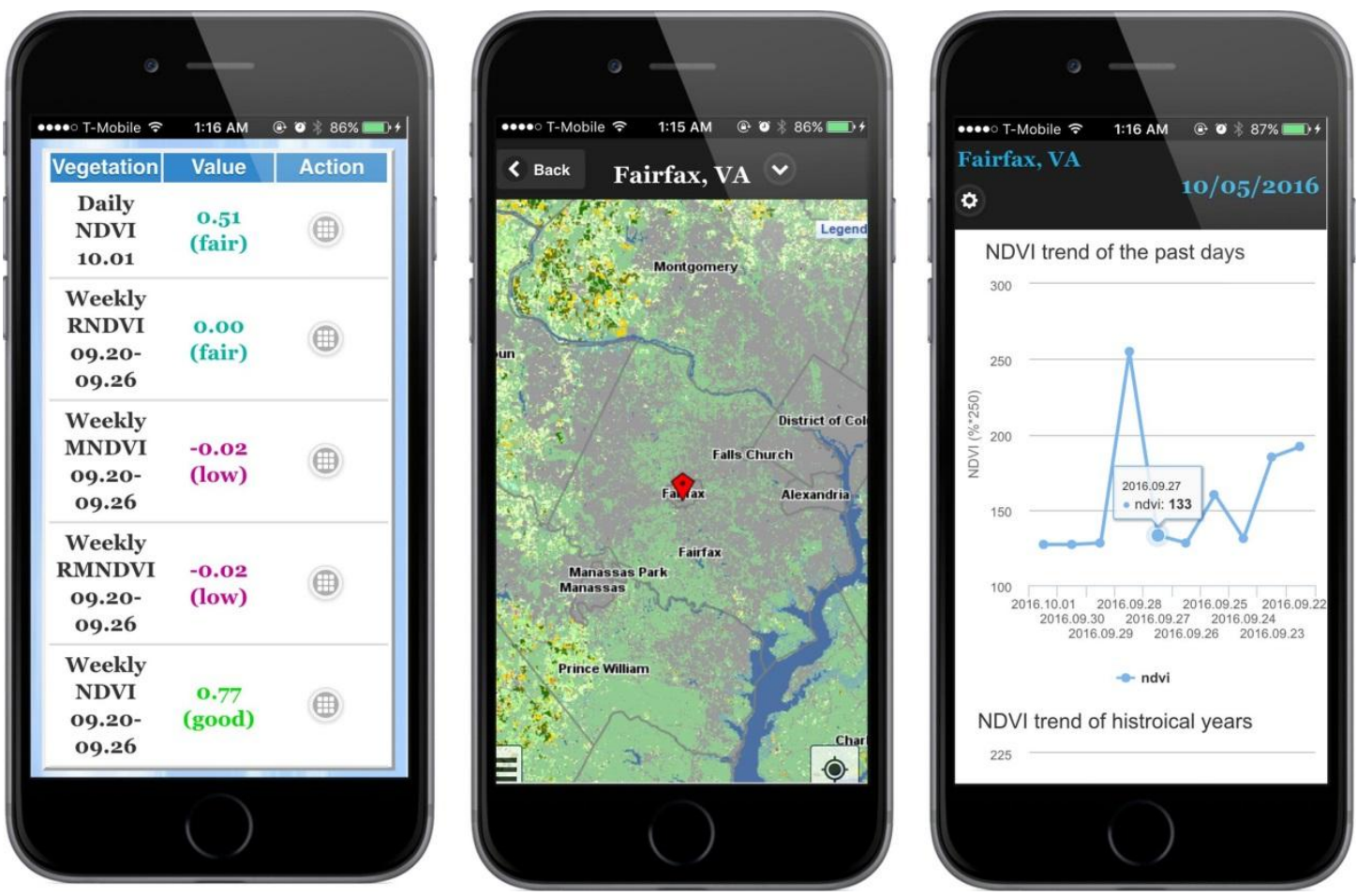

Figure 5. Three view modes (from left to right: table, map and chart)

2) Request generator is used to generate information requests. The requests are encoded by JSON and include coordinates and the required GI names. The requests are submitted to server module via message transceiver. The coordinates can be either the real time locations of mobile devices or specified by users. The default contains all the GI in Table 1. Users also can remove some uninterested GI which will not be retrieved at next time.

3) Message transceiver is used to directly communicate messages with the request handler of server module. This module is responsible for message communication on the client side - sending requests to server module and receiving responses. The transceiver is based on HTTP protocol. For each success response that has a 200 status code in its header, message transceiver will invoke the predefined response parser with the responded content. Otherwise, message transceiver will invoke some fault handlers to deal with the failure and warn users on client interface.

4) Response parser is used to parse the information back from server module. This module simply decodes the information file (JSON), decomposes and pushes the information of different GI into corresponding channels for final exhibition on information viewer. 
5) Location manager is used to manage all the locations viewed by users. GeoFairy supports users to check any location besides their real time locations. For example, a farmer wants to check the GI on his farm while he travels to other places. He could use location manager to find the location of his farm and set it as current location. Client module will invoke the server module with the new location and all the GI in the information viewer will be updated with the responded information.

\section{Implementation}

We implemented a system named GeoFairy for validation. The programming languages, libraries and tools used in GeoFairy include Java, C++, Shell, Javascript, HTML5, CSS3, MySQL, GeoTools, GDAL, Eclipse, Apache Tomcat, Apache HTTP Server, Apache Cordova, iOS SDK, Android SDK and JSON Java processing API. Java is used to develop most components of the server module. GDAL $\mathrm{C}++$ and GeoTools are used in basic image and feature processing such as getting metadata and getting the value of a specific pixel. JSON Java processing API decodes and encodes JSON messages on the server module. Javascript, HTML5 and CSS3 are used in the client module of GeoFairy. MySQL is the database in the server module to record context information and enhance user experiences. Eclipse is used as the integrated development environment. Apache Tomcat is the Web App container. Apache HTTP server provides a Web URL and redirects all the requests to the Tomcat which actually hosts GeoFairy. Apache Cordova creates Mobile Apps independent from particular mobile platforms based on the Javascript, HTML5 and CSS3 files. The biggest advantage of Apache Cordova is that developers only code one time and Cordova can make them be available on almost all the mobile platforms. Cordova could automatically generate a project of mobile SDK. The SDKs are required to generate the final installable mobile App.

The server module of GeoFairy is deployed on instances of a private cloud established by Apache CloudStack. GeoFairy Apps for both iOS platform and Android platform are produced and published on Apple App Store (https://itunes.apple.com/us/app/Geofairy/id1039411946?mt=8) and Google Play Store

(https://play.google.com/store/Apps/details?id=edu.gmu.csiss.mobile.Geofairy\&hl=en ). Anyone could install and use them. Fig. 6 illustrates some screenshots of GeoFairy. 

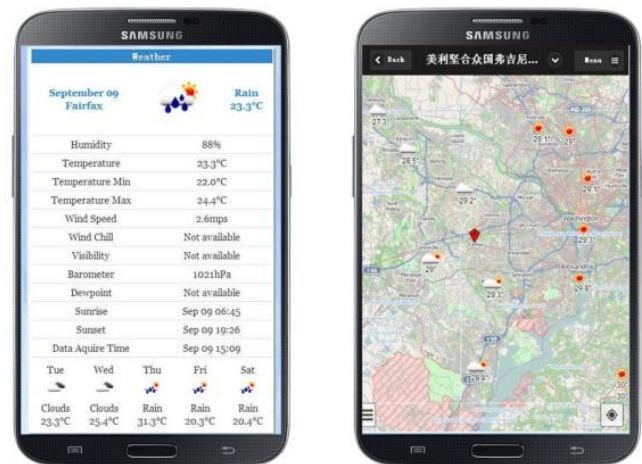

(a)
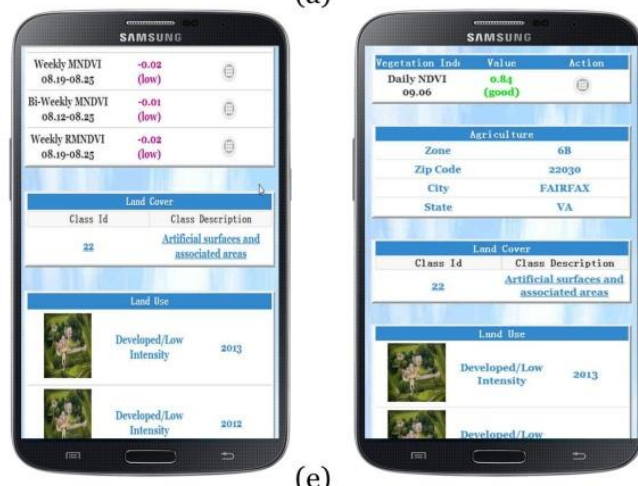

(e)
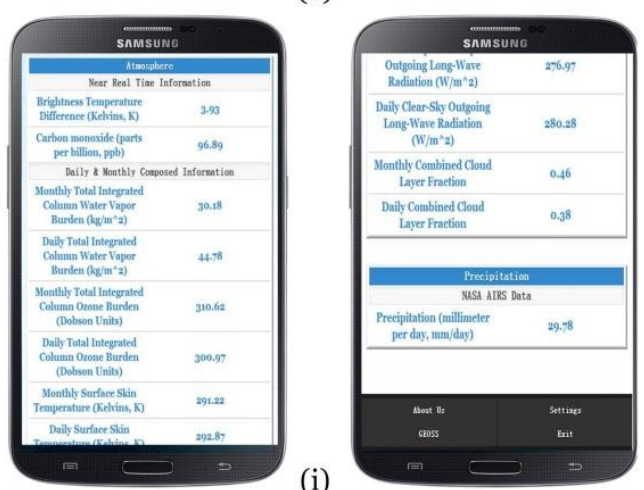

(b)
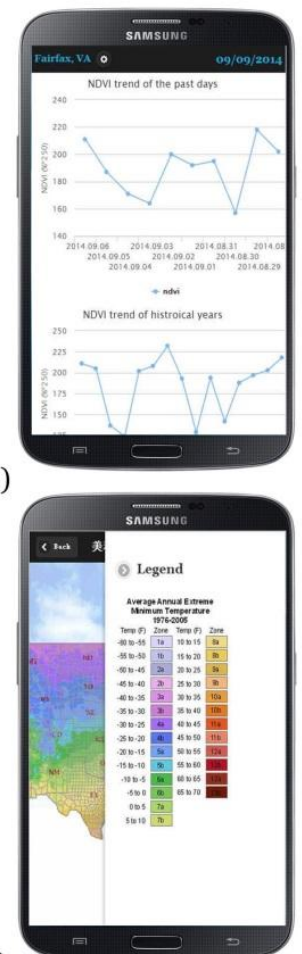

(f)

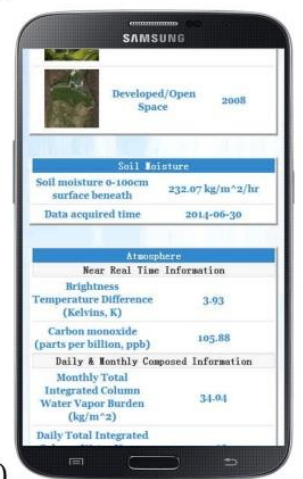

(c)
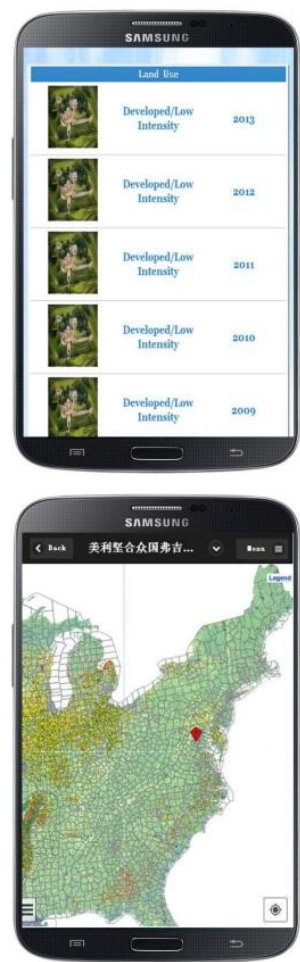

(d)

(g)

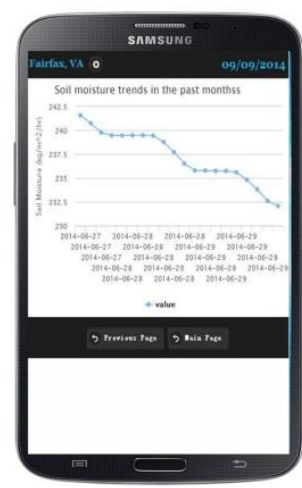

(h)

(1)

Figure 6. Screenshots of GeoFairy. (a) weather table; (b) weather map; (c) meteorology chart; (d) land use table; (e) land cover table; (f) hardiness table; (g) hardiness map with legend; (h) land use map; (i) atmosphere table; (j) precipitation table; (k) soil moisture table; (l) soil moisture chart

\section{Experiments and results}

Experiments have been made to evaluate GeoFairy's coverage, responding speed, precision and consistency. We preliminarily installed GeoFairy on twenty six mobile devices, including eighteen iPhones and eight Samsung Android phones, and use them to retrieve GI. The services they accessed include NOAA weather service, GMU vegetation index products, elevation, Google Geocode service, USDA cropland data layer and hardiness zone product, NASA soil moisture and atmosphere products. The 
test shows the devices can all quickly get correct GI on their real time locations. To more comprehensively reflect the capacity of GeoFairy, we design and conduct two test plans: stress test and multi-location test.

\subsection{Stress Test}

Stress test was conducted on GeoFairy server module to measure the robustness and availability of the server module. First, we used Apache JMeter [54] to design a test plan with one hundred concurrent users (Fig. 7a). Each user corresponds to a thread. Every thread sends a number of HTTP requests for GI of a location to the GeoFairy server. Fig. $7 b$ illustrates part of the test result table. The table records the waiting time between request and response in milliseconds, the status of the response and the message volume. Fig. $7 \mathrm{c}$ shows a sample response data from the NOAA weather service. The overall results are plotted in Fig. 8. There are eight charts displaying eight parameters (from left to right, from top to bottom): average response time, minimum response time, maximum response time, standard deviation response time, error rate, throughput, speed and average bytes. It can be seen that the error rate for all kinds of GI is zero which means all the requests got correct responses even though one hundred users visit at the same time. The average time is less than one second for address and elevation; around five seconds for precipitation, atmosphere, hardiness, vegetation and weather; about ten seconds for land use; about fifteen seconds for soil moisture; about one minute for land cover. The land cover is the slowest because it need return a long list of historical data from multiple years of land cover products. The fastest GI types are the address from Google and the elevation from USGS. The throughput indicates how many requests the server can handle in one second. Larger number represents better performance. Except precipitation, all the GI types have similar throughput. Soil moisture obviously has the fastest delivering speed and the largest average response size. Generally, the average waiting time of some types is a little unendurable as the patience of a user usually won't last longer than seven seconds. Thus, one hundred users are too many for one instance. Load balancer is needed to reduce the load and accelerate the average speed. 


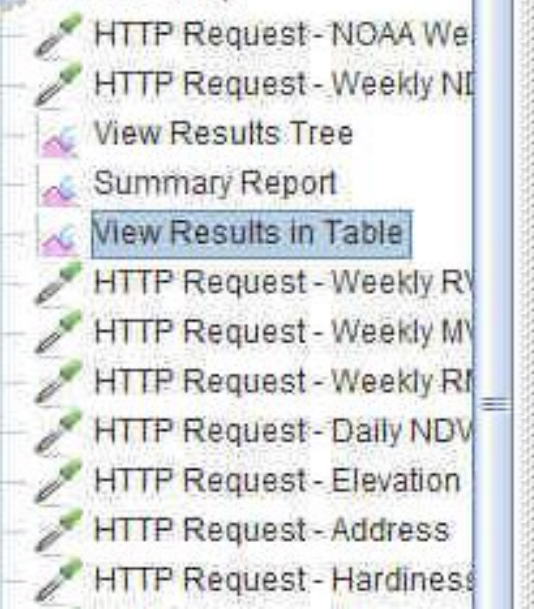

(a)

\begin{tabular}{|c|c|c|c|c|c|c|c|}
\hline Sample \# & Start Time & Thread Name & Label & Sample Time(ms) & Status & Bytes & Latency \\
\hline 1 & $18: 34: 56: 927$ & Thread Group 1-6 & HTTP Request -... & $\begin{array}{r}7956 \\
\end{array}$ & $\theta$ & 5228 & 7956 \\
\hline 2 & $18: 35: 01.473$ & Thread Group 1-15 & HTTP Request -... & 3412 & $\theta$ & 5228 & 3412 \\
\hline 3 & $18: 34: 59.472$ & Thread Group 1-11 & HTTP Request -... & 5414 & $\theta$ & 5228 & 5414 \\
\hline 4 & $18: 35: 02.979$ & Thread Group 1-18 & HTTP Request -... & 1923 & $\theta$ & 5228 & 1923 \\
\hline 5 & $18: 34: 55.454$ & Thread Group 1-3 & HTTP Request -... & 9447 & $\theta$ & 5228 & 9447 \\
\hline 6 & $18: 34: 55.950$ & Thread Group 1-4 & HTTP Request -... & 8955 & $\theta$ & 5228 & 8955 \\
\hline 7 & $18: 35: 03.485$ & Thread Group 1-19 & HTTP Request -... & 1422 & $\theta$ & 5228 & 1422 \\
\hline 8 & $18: 35: 03.960$ & Thread Group 1-20 & HTTP Request -... & 951 & $\theta$ & 5228 & 951 \\
\hline 9 & $18: 34: 59.957$ & Thread Group 1-12 & HTTP Request -... & 4955 & 8 & 5228 & 4955 \\
\hline 10 & $18: 34: 57.464$ & Thread Group 1-7 & HTTP Request -... & 7480 & $\theta$ & 5228 & 7480 \\
\hline 11 & $18: 3458.930$ & Thread Group 1-10 & HTTP Request -... & 6015 & $\theta$ & 5228 & 6015 \\
\hline 12 & $18: 34.54 .923$ & Thread Group 1-2 & HTTP Request -... & 10032 & $\theta$ & 5228 & 10032 \\
\hline 13 & $18: 34: 57.954$ & Thread Group 1-8 & HTTP Request -... & 7140 & $\theta$ & 5228 & 7140 \\
\hline 14 & $18: 35: 01.959$ & Thread Group 1-16 & HTTP Request -... & 3143 & $\theta$ & 5228 & 3143 \\
\hline 15 & $18: 34: 54.417$ & Thread Group 1-1 & HTTP Request -... & 10698 & $\theta$ & 5228 & 10698 \\
\hline 16 & $18: 35: 00.532$ & Thread Group 1-13 & HTTP Request -... & 4614 & $\theta$ & 5228 & 4614 \\
\hline 17 & $18: 35: 00.975$ & Thread Group 1-14 & HTTP Request -... & 4193 & $\theta$ & 5228 & 4193 \\
\hline 18 & $18: 34: 58.534$ & Thread Group 1-9 & HTTP Request -... & 6935 & $\theta$ & 5228 & 6935 \\
\hline 19 & $18: 35: 04.535$ & Thread Group 1-21 & HTTP Request -... & 978 & $\theta$ & 5228 & 978 \\
\hline 20 & $18: 35: 05.486$ & Thread Group 1-23 & HTTP Request -... & 551 & $\theta$ & 5228 & 551 \\
\hline 21 & $18: 35: 05.961$ & Thread Group 1-24 & HTTP Request -... & 332 & $\theta$ & 5228 & 332 \\
\hline 22 & $18: 35: 06.536$ & Thread Group 1-25 & HTTP Request -... & 565 & $\theta$ & 5228 & 555 \\
\hline
\end{tabular}

(b) 
(c)

Figure 7. JMeter stress test interface on single instance. (a) designed HTTP requests; (b) test monitoring results; (c) NOAA weather response data 

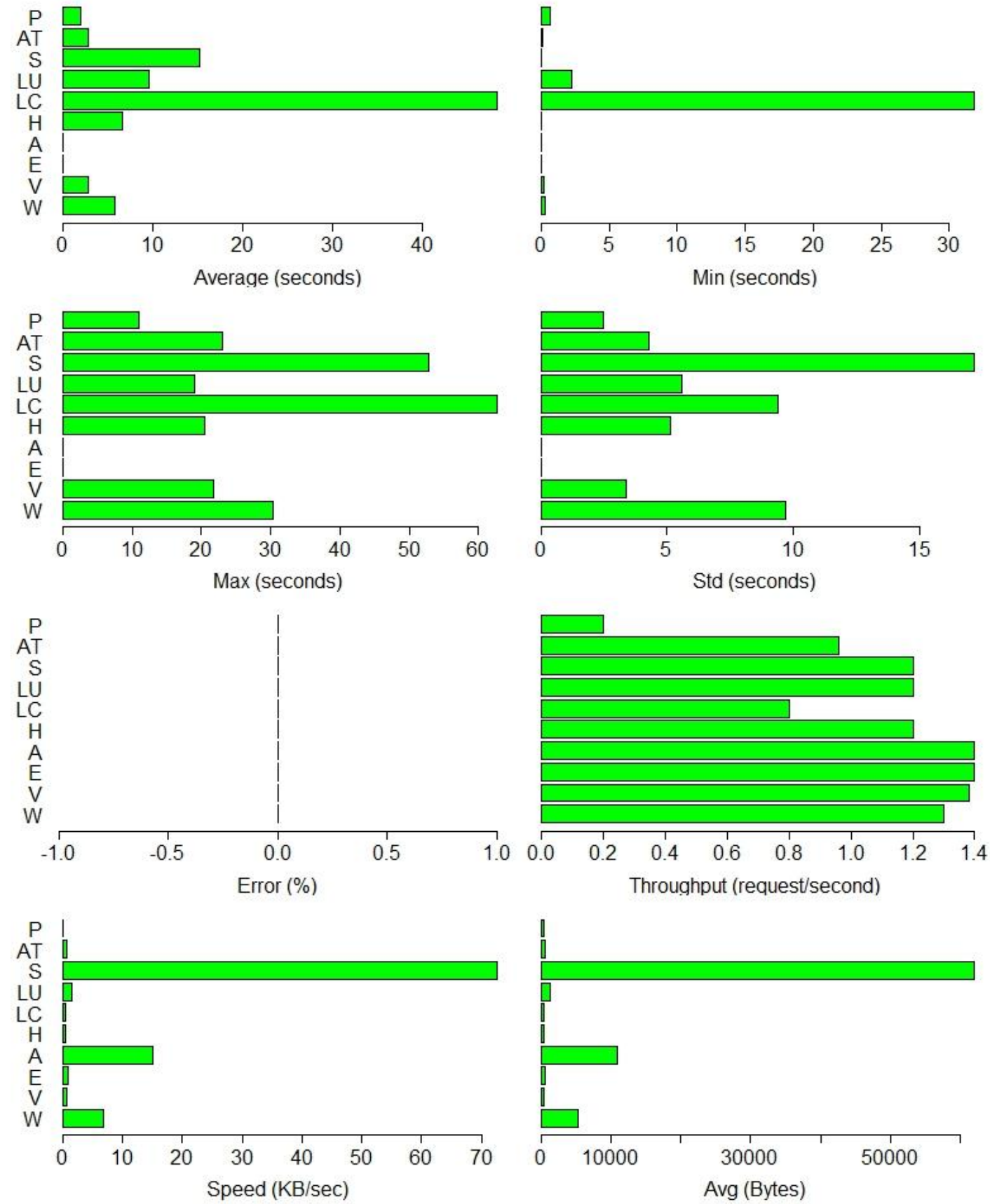

Figure 8. Charts of the stress test results on single instance (P: Precipitation; AT: Atmosphere; S: Soil Moisture; LU: Land Use; LC: Land Cover; H: Hardiness; A: Address; E: Elevation; V: Vegetation; W: Weather)

\subsection{Multi-Location Test}

With load balancer enabled, we made one hundred concurrent thread tests on ten scattered locations listed in Table 3. Most products used in GeoFairy spatially cover the globe, except the cropland data layer and the hardiness zone product. The test 
locations are mainly in the United States as it has the best coverage. Fig. 9 visualizes the average response time for the ten locations, The U.S. domestic locations are shown in Fig. 9a while the outside U.S. locations are rendered in Fig. 9b. It is obvious that the average response time is under two seconds which are completely endurable for most users. This test proves that with load balancer enabled, GeoFairy is capable of one-stop gathering and delivering distributed GI from different providers to globally scattered mobile endpoint devices based on their real time locations in an endurable speed and zero error rate.

Table 3. Test locations

\begin{tabular}{ccc}
\hline Latitude, Longitude & City & Country \\
\hline $38.845259,-77.306387$ & Fairfax, VA & U.S.A. \\
$40.771566,-73.974837$ & New York & U.S.A. \\
$42.363112,-71.060885$ & Boston & U.S.A. \\
$40.763333,-111.894131$ & Salt Lake City & U.S.A \\
$33.912747,-118.260960$ & Los Angeles & U.S.A. \\
$25.765577,-80.195346$ & Miami & U.S.A. \\
$39.901277,116.403439$ & Beijing & China \\
$51.519099,-0.141213$ & London & U.K. \\
$-26.199458,28.042025$ & Johannesburg & South Africa \\
$35.706898,139.738161$ & Tokyo & Japan \\
\hline
\end{tabular}

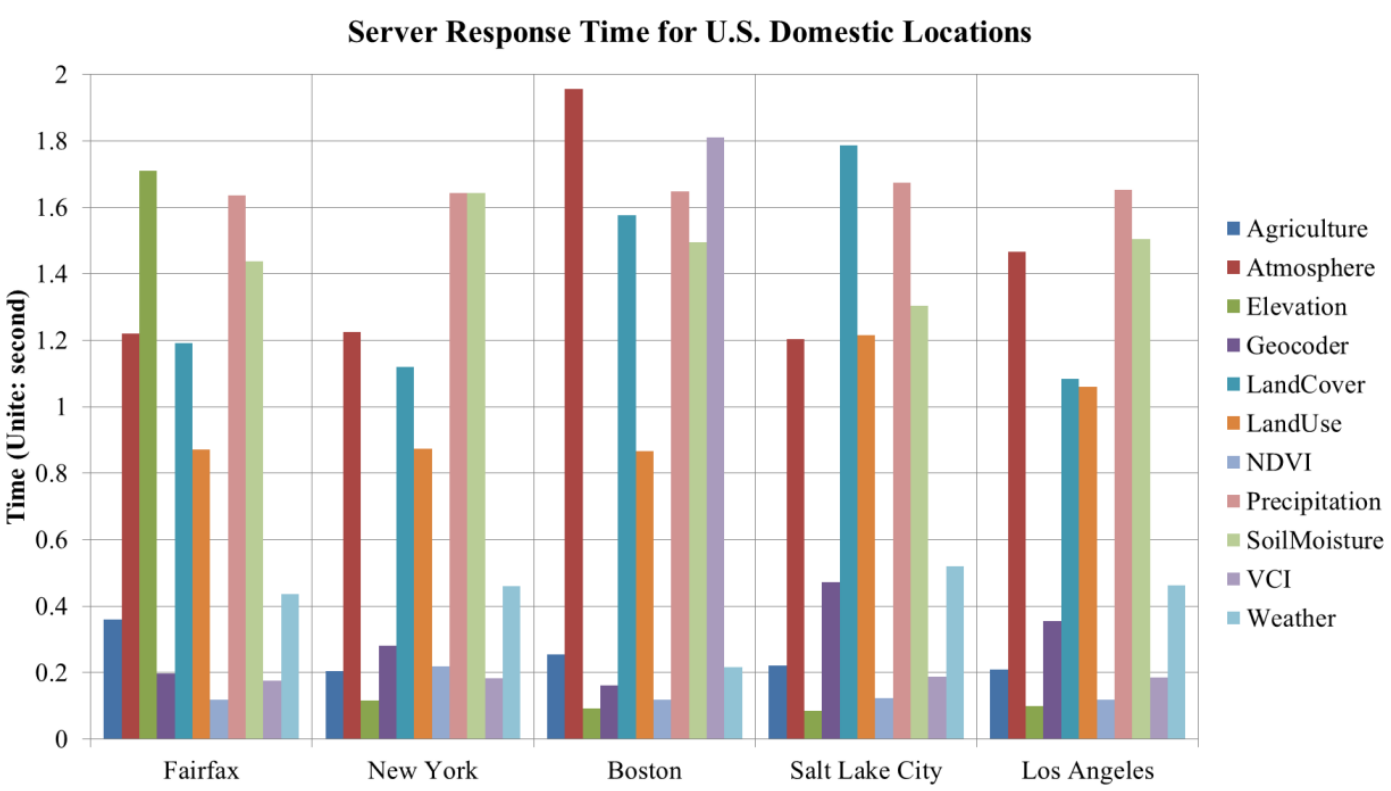

(a) 


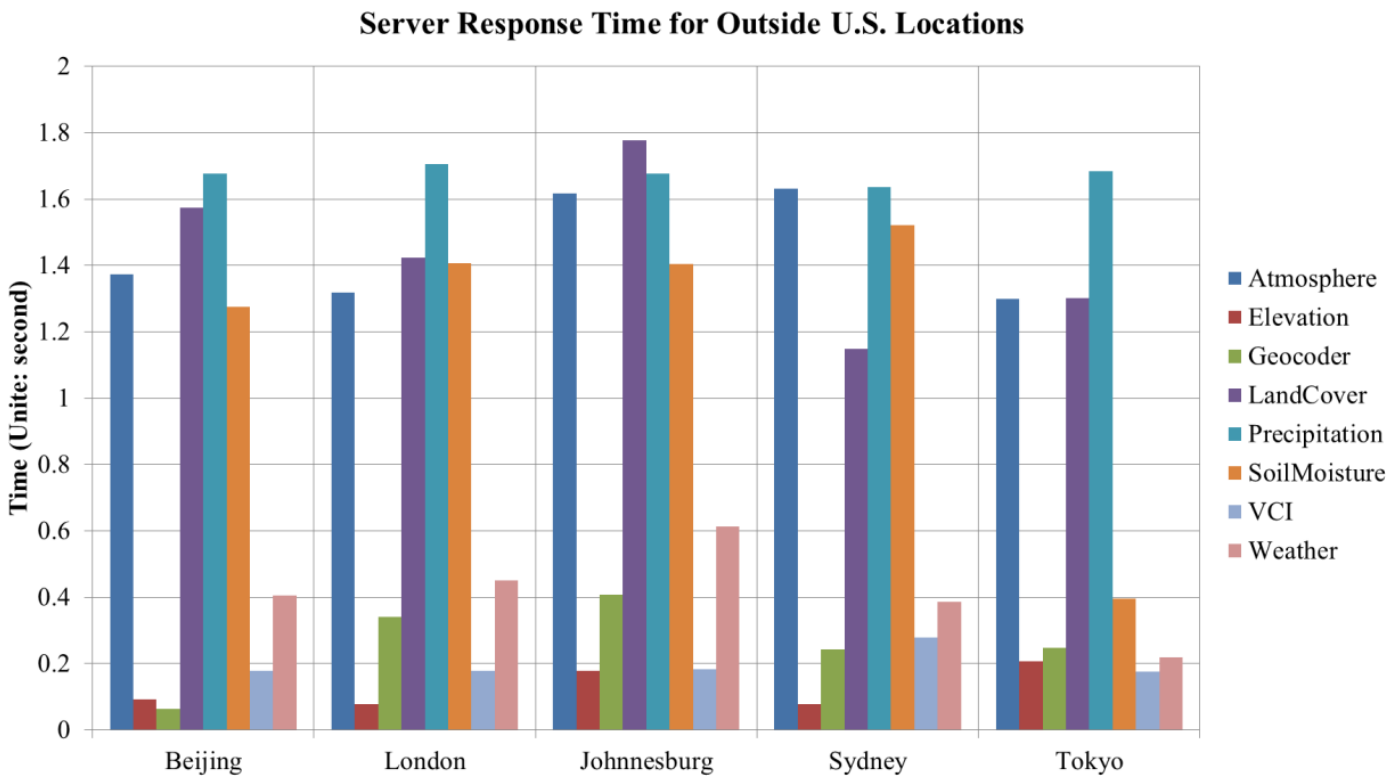

(b)

Figure 9. Average response time of location enabled load balancer

\section{Discussion}

\subsection{What is new?}

The basic techniques are not newly created. The original contribution is the GeoFairy framework which is able to smoothly plug in the existing techniques and apply them on distributed and heterogeneous geospatial resources. The integration successfully achieves the goal of on-demand gathering from distributed providers and delivering twelve kinds of specialized GI in a genuine one-stop and location based manner. Compared to the existing geoportals which only serves metadata catalogs, the one-stop service on-demand invokes the distributed services, directly returns twelve kinds of GI and the content is customized by users' real time locations. Such a service has never been realized before.

\subsection{Location Enabled Balancer}

A LBS will receive a great amount of parallel requests when the number of users increases to a large scale. Different from the other services, LBS usually has a cache mechanism to accelerate the loading speed for user intensive locations, such as downtown areas in major cities. However, traditional load balancing algorithms do not consider user location when forwarding requests to instance servers. It is a waste of system resources and will result in bad user experiences if the server with cache 
cannot process corresponding requests. Thus, a location enabled load balancing algorithm is developed. The new algorithm takes user location into account and can facilitate the data cache mechanism and prevent the server module from over loading. The servers with cache will have higher weights in request assignment. This algorithm can benefit all the LBS configured with load balancers.

\subsection{Instant Benefit}

The short-term benefits of GeoFairy are very obvious such as time saving and convenience. Users can get GI of a location with no need to spend time on any of searching, researching, downloading and extracting. GeoFairy enables wireless information retrieval on the fields via mobile platforms so that users can access GI anywhere and anytime. The delivery is straight forward. GI values directly pop up when the App is opened. The functionality is ideal for consumers unwilling to deal with professional actions and terms about GI.

\subsection{Long Term Benefit}

From a long term perspective, GeoFairy significantly lowers the barrier of entry to GI and fills the gap between specialized GI and general users. In GeoFairy, GI is automatically retrieved, processed and transferred from data sources to end users. All the middle steps are automatically completed via machine-to-machine communication. The technical details are hidden and user prerequisite is mostly removed. General users can directly access the GI with no need to learn any knowledge and techniques of GI and web services. It can be expected that GeoFairy will be very useful in many real world scenarios such as quick daily GI inquiry, environmental agency decision making support, disaster monitoring and responding, agricultural decision making assistance, meteorology monitoring and forecast, city planning, forest management, etc.

\subsection{Disadvantages}

At present, GeoFairy is unable to support offline operation and the locations without network connections cannot use GeoFairy. The server module is responsible for most of the heavy duty processing tasks and the GI is real-time and on-demand gathered from remote providers. So good networking, either wired or wireless, is essential to maintain the availability of GeoFairy. In some ultimate situations like earthquakes which can cause network interruption, GeoFairy can barely serve in the present form.

\section{Conclusion and future work}


This paper proposes a framework to realize one-stop and global LBS for GI retrieval on mobile platforms. The framework originally integrates a number of state-of-the-art techniques with geospatial resources and let them cooperate together to provide a robust and highly available LBS. Cloud platform is used to deploy the server module. A location enabled load balancing algorithm is presented to balance the cloud instance VMs on behalf of LBS requirements. A system named GeoFairy is developed to implement the framework. It provides a genuine one-stop service for gathering and delivering twelve kinds of GI on real time location. Two Apps are built for the major mobile ecosystems: iOS and Android. Many tests, including stress test, have been made on GeoFairy via a number of mobile devices at various locations. The results demonstrate that GeoFairy is capable of one-stop delivering real-time GI to users and significantly reducing costs for users on information searching and retrieving. This feature is very helpful in emergent scenarios such as disaster responding and military actions. This research paves the way on both theoretical and practical aspects for researchers and developers to realize operational mobile applications for one stop and location based GI retrieval.

In future, improvements will be made on the server module to serve more and more GI. Both the spatial and temporal coverage of GI will be enhanced to a high resolution. The processing efficiency of the server module will be further improved by leveraging software level solutions such as Hadoop and Spark. In addition, the client module will be augmented with data cache mechanism to enable offline operation.

\section{Reference}

[1] D. C. Cox, "Wireless network access for personal communications," Communications Magazine, IEEE, vol. 30, pp. 96-115, 1992.

[2] W. Lehr and L. W. McKnight, "Wireless internet access: 3G vs. WiFi?," Telecommunications Policy, vol. 27, pp. 351-370, 2003.

[3] M. A. Visser and M. El Zarki, "Voice and data transmission over an 802.11 wireless network," in Personal, Indoor and Mobile Radio Communications, 1995. PIMRC'95. Wireless: Merging onto the Information Superhighway., Sixth IEEE International Symposium on, 1995, pp. 648-652.

[4] D. Tang and M. Baker, "Analysis of a local-area wireless network," in Proceedings of the 6th annual international conference on Mobile computing and networking, 2000, pp. 1-10.

[5] E. Oh, B. Krishnamachari, X. Liu, and Z. Niu, "Toward dynamic energy-efficient operation of cellular network infrastructure," Communications Magazine, IEEE, vol. 49, pp. 56-61, 2011.

[6] H. S. Park, S. H. Yoon, T. H. Kim, J. S. Park, M. S. Do, and J. Y. Lee, "Vertical Hando. Procedure and Algorithm between IEEE802. 11 WLAN and CDMA Cellular Network," in Mobile Communications, ed: Springer, 2003, pp. 103-112.

[7] T. S. Rappaport, Wireless communications: principles and practice vol. 2: Prentice Hall PTR 
New Jersey, 1996.

[8] R. Ballagas, J. Borchers, M. Rohs, and J. G. Sheridan, "The smart phone: a ubiquitous input device," Pervasive Computing, IEEE, vol. 5, pp. 70-77, 2006.

[9] D. Williams, Corona sdk application design: Packt Publishing Ltd, 2013.

[10] J. M. Wargo, Apache Cordova 4 Programming: Pearson Education, 2015.

[11] J. M. Wargo, PhoneGap Essentials: Building Cross-Platform Mobile Apps: Addison-Wesley, 2012.

[12] T. Hey and A. E. Trefethen, "Cyberinfrastructure for e-Science," Science, vol. 308, pp. 817-21, May 62005.

[13] C. Council, Cyberinfrastructure vision for 21st century discovery: National Science Foundation, Cyberinfrastructure Council, 2007.

[14] S. Wang, "A CyberGIS framework for the synthesis of cyberinfrastructure, GIS, and spatial analysis," Annals of the Association of American Geographers, vol. 100, pp. 535-557, 2010.

[15] D. J. Wright and S. Wang, "The emergence of spatial cyberinfrastructure," Proceedings of the National Academy of Sciences, vol. 108, pp. 5488-5491, 2011.

[16] C. Yang, R. Raskin, M. Goodchild, and M. Gahegan, "Geospatial cyberinfrastructure: past, present and future," Computers, Environment and Urban Systems, vol. 34, pp. 264-277, 2010.

[17] M. A. Brovelli, H. Mitasova, M. Neteler, and V. Raghavan, "Free and open source desktop and Web GIS solutions," Applied Geomatics, vol. 4, pp. 65-66, 2012.

[18] R. Wolfe, "MODIS Web Services Synchronous Post-processing Approach," in AGU Fall Meeting Abstracts, 2010, p. 1390.

[19] A. Chen, G. Leptoukh, S. J. Kempler, and L. Di, "Visualization of NASA Earth science data in Google Earth," in Sixth International Conference on Advanced Optical Materials and Devices, 2008, pp. 714329-714329-13.

[20] K. Ward, "NASA Earth Observations (NEO): Data imagery for education and visualization," in AGU Fall Meeting Abstracts, 2008, p. 03.

[21] R. Fielding, "Representational state transfer," Architectural Styles and the Design of Netowork-based Software Architecture, pp. 76-85, 2000.

[22] C. Pautasso, O. Zimmermann, and F. Leymann, "Restful web services vs. big'web services: making the right architectural decision," in Proceedings of the 17th international conference on World Wide Web, 2008, pp. 805-814.

[23] P. Mazzetti and S. Nativi, "RESTful Implementation of Geospatial Services," $E G U$ Geophysical Research Abstracts, vol. 10, 2008.

[24] G. Percivall, "Progress in ogc web services interoperability development," in Standard-Based Data and Information Systems for Earth Observation, ed: Springer, 2010, pp. 37-61.

[25] E. Kaplan and C. Hegarty, Understanding GPS: principles and applications: Artech house, 2005.

[26] V. Otsason, A. Varshavsky, A. LaMarca, and E. De Lara, "Accurate GSM indoor localization," in International conference on ubiquitous computing, 2005, pp. 141-158.

[27] H. Koyuncu and S. H. Yang, "A survey of indoor positioning and object locating systems," IJCSNS International Journal of Computer Science and Network Security, vol. 10, pp. $121-128,2010$

[28] J. Schiller and A. Voisard, Location-based services: Elsevier, 2004

[29] A. Küpper, Location-based services: fundamentals and operation: John Wiley \& Sons, 2005. 
[30] I. Satoh, "Location-based services in ubiquitous computing environments," International Journal on Digital Libraries, vol. 6, pp. 280-291, 2006.

[31] K. Virrantaus, J. Markkula, A. Garmash, V. Terziyan, J. V. Alainen, A. Katanosov, and H. Tirri, "Developing GIS-supported location-based services," in Web information systems engineering, 2001. Proceedings of the Second International Conference on, 2001, pp. 66-75.

[32] B. Jiang and X. Yao, "Location-based services and GIS in perspective," Computers, Environment and Urban Systems, vol. 30, pp. 712-725, 2006.

[33] M. Armbrust, A. Fox, R. Griffith, A. D. Joseph, R. Katz, A. Konwinski, G. Lee, D. Patterson, A. Rabkin, and I. Stoica, "A view of cloud computing," Communications of the ACM, vol. 53, pp. 50-58, 2010.

[34] P. Mell and T. Grance, "The NIST definition of cloud computing," ed. Special Publication (NIST SP) - 800-145, 2011.

[35] O. Sefraoui, M. Aissaoui, and M. Eleuldj, "OpenStack: toward an open-source solution for cloud computing," International Journal of Computer Applications, vol. 55, 2012.

[36] R. Kumar, K. Jain, H. Maharwal, N. Jain, and A. Dadhich, "Apache cloudstack: Open source infrastructure as a service cloud computing platform," Proceedings of the International Journal of advancement in Engineering technology, Management and Applied Science, pp. 111-116, 2014.

[37] D. Nurmi, R. Wolski, C. Grzegorczyk, G. Obertelli, S. Soman, L. Youseff, and D. Zagorodnov, "The eucalyptus open-source cloud-computing system," in Cluster Computing and the Grid, 2009. CCGRID'09. 9th IEEE/ACM International Symposium on, 2009, pp. 124-131.

[38] D. Milojičić, I. M. Llorente, and R. S. Montero, "OpenNebula: A cloud management tool," IEEE Internet Computing, pp. 11-14, 2011.

[39] M. G. Tait, "Implementing geoportals: applications of distributed GIS," Computers, Environment and Urban Systems, vol. 29, pp. 33-47, 2005.

[40] D. J. Maguire and P. A. Longley, "The emergence of geoportals and their role in spatial data infrastructures," Computers, Environment and Urban Systems, vol. 29, pp. 3-14, 2005.

[41] M. F. Goodchild, P. Fu, and P. Rich, "Sharing geographic information: an assessment of the Geospatial One-Stop," Annals of the Association of American Geographers, vol. 97, pp. 250-266, 2007.

[42] L. Bernard, I. Kanellopoulos, A. Annoni, and P. Smits, "The European geoportal—one step towards the establishment of a European Spatial Data Infrastructure," Computers, Environment and Urban Systems, vol. 29, pp. 15-31, 2005.

[43] G. Giuliani and P. Peduzzi, "The PREVIEW Global Risk Data Platform: a geoportal to serve and share global data on risk to natural hazards," Natural Hazards and Earth System Science, vol. 11, pp. 53-66, 2011.

[44] S. Roche, E. Propeck-Zimmermann, and B. Mericskay, "GeoWeb and crisis management: issues and perspectives of volunteered geographic information," GeoJournal, vol. 78, pp. 21-40, 2013.

[45] C. M. Croner, "Public health, GIS, and the Internet," Annual Review of Public Health, vol. 24, pp. 57-82, 2003.

[46] C. Weidemann and J. Swift, "Social Media Location Intelligence: The Next Privacy Battle-An ArcGIS add-in and Analysis of Geospatial Data Collected from Twitter. com," International Journal of Geoinformatics, vol. 9, 2013. 
[47] H. Samet, B. C. Fruin, and S. Nutanong, "Duking it out at the smartphone mobile App mapping API corral: Apple, Google, and the competition," presented at the Proceedings of the First ACM SIGSPATIAL International Workshop on Mobile Geographic Information Systems, Redondo Beach, California, 2012.

[48] G. Goggin, F. Martin, and T. Dwyer, "Locative News," Journalism Studies, vol. 16, pp. 41-59, 2015/01/02 2015.

[49] H.-M. Chuang, C.-H. Chang, T.-Y. Kao, C.-T. Cheng, Y.-Y. Huang, and K.-P. Cheong, "Enabling maps/location searches on mobile devices: constructing a POI database via focused crawling and information extraction," International Journal of Geographical Information Science, vol. 30, pp. 1405-1425, 2016/07/02 2016.

[50] C. Becker and C. Bizer, "Exploring the Geospatial Semantic Web with DBpedia Mobile," Web Semantics: Science, Services and Agents on the World Wide Web, vol. 7, pp. 278-286, 2009.

[51] J. Yin and J. D. Carswell, "Touch2Query enabled mobile devices: a case study using OpenStreetMap and iPhone," in International Symposium on Web and Wireless Geographical Information Systems, 2011, pp. 203-218.

[52] J. Bao, Y. Zheng, and M. F. Mokbel, "Location-based and preference-aware recommendation using sparse geo-social networking data," presented at the Proceedings of the 20th International Conference on Advances in Geographic Information Systems, Redondo Beach, California, 2012.

[53] R. Abdalla, "Mobile GIS and Location-Based Services (LBS)," in Introduction to Geospatial Information and Communication Technology (GeoICT), ed Cham: Springer International Publishing, 2016, pp. 83-103.

[54] E. H. Halili, Apache JMeter: A practical beginner's guide to automated testing and performance measurement for your websites: Packt Publishing Ltd, 2008. 